\title{
Single item measures of self-rated mental health: a scoping review
}

\author{
Farah Ahmad ${ }^{1 *}$, Anuroop K Jhajj ${ }^{2}$, Donna E Stewart ${ }^{3}$, Madeline Burghardt ${ }^{4}$ and Arlene S Bierman ${ }^{5}$
}

\begin{abstract}
Background: A single-item measure of self-rated mental health (SRMH) is being used increasingly in health research and population health surveys. The item asks respondents to rate their mental health on a five-point scale from excellent to poor. This scoping study presents the first known review of the SRMH literature.

Methods: Electronic databases of Medline, CINAHL, PsycINFO, EMBASE and Cochrane Reviews were searched using keywords. The databases were also searched using the titles of surveys known to include the SRMH single item. The search was supplemented by manually searching the bibliographic sections of the included studies. Two independent reviewers coded articles for inclusion or exclusion based on whether articles included SRMH. Each study was coded by theme and data were extracted about study design, sample, variables, and results.

Results: Fifty-seven studies included SRMH. SRMH correlated moderately with the following mental health scales: Kessler Psychological Distress Scale, Patient Health Questionnaire, mental health subscales of the Short-Form Health Status Survey, Behaviour and Symptom Identification Scale, and World Mental Health Clinical Diagnostic Interview Schedule. However, responses to this item may differ across racial and ethnic groups. Poor SRMH was associated with poor self-rated health, physical health problems, increased health service utilization and less likelihood of being satisfied with mental health services. Poor or fair SRMH was also associated with social determinants of health, such as low socioeconomic position, weak social connections and neighbourhood stressors. Synthesis of this literature provides important information about the relationships SRMH has with other variables.
\end{abstract}

Conclusions: SRMH is associated with multi-item measures of mental health, self-rated health, health problems, service utilization, and service satisfaction. Given these relationships and its use in epidemiologic surveys, SRMH should continue to be assessed as a population health measure. More studies need to examine relationships between SRMH and clinical mental illnesses. Longitudinal analyses should look at whether SRMH is predictive of future mental health problems.

Keyword: Self rated mental health, Single item, Review, Measurement

\section{Background}

Short scales to measure physical and mental health of populations are increasingly used in epidemiologic surveys [1] to reduce respondent burden and simplify administration and translation while providing efficient global health indicators. The use of single-item measures is also on the rise. This includes the measure of selfrated mental health (SRMH): "In general, would you say your mental health is: Excellent, Very Good, Good, Fair or Poor?"

\footnotetext{
* Correspondence: farahmad@yorku.ca

'School of Health Policy and Management, Faculty of Health, York University, 4700 Keele Street, HNES 414, Ontario M3J 1P3, Canada

Full list of author information is available at the end of the article
}

The early use of the single SRMH item includes studies conducted in 1970s with college students in relation to personality traits and help-seeking for mental health [2,3]. In 1981 it was used as part of the National Institute of Health Diagnostic Interview Schedule, which was developed using criteria from the Diagnostic and Statistical Manual of Mental Disorders-III [4]. A single SRMH item was later included in the World Mental Health Composite International Diagnostic Interview [5]. More recently, the SRMH item has been used as a stand-alone indicator of mental health in small and large scale studies. Examples of the national epidemiologic surveys with SRMH as a stand-alone item are the Canadian Community 
Health Survey and Medical Expenditure Panel Survey $[6,7]$. Researchers report significant relationships between SRMH and mental disorders [8], need for care [9], utilization patterns [10] and adherence to treatment plans [11]. Others have examined its relationship with validated clinical measures for diagnosis of mental health conditions $[8,12]$. In some instances the SRMH item is used as a construct to validate another mental health measure $[13,14]$.

A similar single item of self-rated health (SRH) has been used world-wide since the 1950s [15]. SRH asks individuals to rate their health on a 5 -point scale ranging from excellent to poor, as in the SRMH. SRH is a strong predictor of mortality [16], health care utilization $[17,18]$, and morbidity [19]. The parallel wording structure of both items implies that SRMH could potentially measure aspects of mental health as robustly as the physical health indications provided by SRH. Though SRMH is increasingly being used as an indicator of population mental health and as a measure to assess risk for adverse mental health outcomes, less is known about the performance of this item.

Understanding SRMH is important to be able to evaluate previous studies using the item, for application of SRMH as a health indicator, and to provide a basis for future work. Since to our knowledge there are no reviews of SRMH, we initiated a traditional systematic review but the heterogeneity of the published studies using SRMH item didn't allow us to proceed. Thus, we conducted a scoping study of published studies that have either used or analyzed SRMH. The methodology was informed by the scoping review framework developed by Arksey and O'Malley in 2005 [20]. A scoping review or study is conducted to explore and summarize empirical knowledge in a diverse or heterogeneous area of research. Its primary objective is to produce a descriptive overview of research findings. Thus, the goal of this review was to gain a better understanding of how single item SRMH is used in research and how it correlates with other measures and health outcomes in order to inform the use of this measure in population and public health, health systems improvement, and research.

\section{Methods}

\section{Search strategy}

We searched Medline, CINAHL, PsycINFO, EMBASE and Cochrane Reviews from their inception to July 2012. In consultation with a librarian, a broad search strategy was established using keywords as 'free terms'. Keyword searches were conducted on titles and abstracts for any of the following word combinations: self rated mental, self perceived mental, self assessed mental, self reported mental or global mental. The Medline search used following string: ((self adj (reported or assessed or rated or perceived) adj mental) or global mental).tw. An additional strategy searched the aforementioned databases using the keywords mental health along with titles of surveys known to use the item: Canadian Community Health Survey (CCHS), Epidemiologic Catchment Area study (ECA), Medical Expenditure Panel Survey (MEPS), National Latino and Asian American Study (NLAAS), National Health Services Postal Questionnaire, Mexican American Prevalence and Services Study (MAPSS), Ontario Health Survey (OHS) Mental Health Supplement, and the World Mental Health Composite International Diagnostic Interview (WMH-CIDI). Manual searches were conducted using the reference sections of identified articles. The search was restricted to English language articles. We did not search grey literature.

\section{Eligibility criteria}

Articles qualified for inclusion if they assessed self-rated mental health, self-reported mental health, self assessed mental health, self perceived mental health, or individual global mental health. The study needed to contain a single item asking for a general mental health rating on a four or five point scale, and results related to this item. Acceptable variations of this question asked about overall mental health, mental health at the present time, mental health, and emotional health (Table 1). Articles were excluded if SRMH was measured using multiple items, or if the single item asked about a specific disorder or a mental health symptom (e.g. depression or worry or downhearted-and-blue) [21].

We included articles with primary data collection and secondary data analyses. Qualitative studies, literature reviews, and meta-analyses were included but no additional relevant studies were found. The search encompassed all international English articles, and no exclusions were made by sex, gender, ethnicity, geographic location, or age.

\section{Selection process}

Two independent reviewers (FA and $\mathrm{AJ}$ ) considered abstracts for inclusion or exclusion. A coding scheme was established based on study objectives and the first 15 abstracts in the database. The scheme was pilot tested on the first 30 articles and reviewers achieved 100\% consensus. Remaining abstracts were evenly divided for coding. Forty randomly selected abstracts were coded by both reviewers to calculate inter-rater reliability $(\kappa=0.80)$. All articles that met the inclusion criteria were considered relevant and retrieved for further review. In the review of full-articles, studies were classified by their objective for using SRMH in a validation study or other studies, which were grouped according to the use of SRMH as a major variable or a minor variable. SRMH was considered a major variable if it was an outcome variable or one of the principal independent variables. Conversely, SRMH was considered a minor variable if it was included as a 


\begin{tabular}{|c|c|}
\hline Item wording & Source(s) \\
\hline $\begin{array}{l}\text { In general, would you say your mental } \\
\text { health is: }\end{array}$ & MEPS, CCHS \\
\hline \multicolumn{2}{|l|}{ 1. Excellent } \\
\hline \multicolumn{2}{|l|}{ 2. Very Good } \\
\hline \multicolumn{2}{|l|}{ 3. Good } \\
\hline \multicolumn{2}{|l|}{ 4. Fair } \\
\hline 5. Poor & \\
\hline
\end{tabular}

How would you rate your overall mental health?
1. Excellent
2. Very Good
3. Good
4. Fair
5. Poor

At the present time, would you say your emotional health is:
1. Excellent
2. Very Good
3. Good
4. Fair
5. Poor

How do you rate your mental health at the present time?
1. Excellent
2. Good
3. Fair
5. Poor

How do you rate your mental health?
1. Poor
2. Fair
3. Good
4. Very good
5. Excellent

CCHC: Canadian Community Health Survey.

ECA: Epidemiologic Catchment Area.

MEPS: Medical Expenditure Panel Survey.

OHS: Ontario Health Survey.

WMH-CIDI: World Mental Health Composite International Diagnostic Interview.

covariate in models without being discussed in much detail, or if it was not a primary focus in descriptive studies. Studies classified in the minor group [14,17,22-35] were tabulated but excluded from synthesis.

\section{Data synthesis}

We extracted information about each study's design, country of origin, data source, and sample characteristics (sample size, gender distribution, age range, population type and ethnicity). We identified variables that were studied in relation to SRMH, and isolated the results of these analyses. A thematic analysis was conducted through review and re-review of the included articles by the research team until group consensus was achieved. We identified following SRMH research themes: validation; mental health conditions; physical health; use of health services; and social determinants of health. These categories were not mutually exclusive, and some studies fit under multiple themes (e.g. $[9,36]$ ).

\section{Results}

The literature search found 1271 unique abstracts, 130 of which qualified for full-text review. Thirty-seven articles qualified for inclusion and seventeen new additions were found by searching databases by scales known to contain the SRMH item. Three more articles were found by searching reference sections, for a total of 57 relevant articles. Figure 1 describes the literature review and search process. Please see Additional file 1 for the 57 identified studies.

\section{Study characteristics}

Table 2 presents overall study characteristics. While first study on SRMH was published in 1980, additional studies didn't appear until 1997. SRMH use in research appears to have increased since 2004 (Figure 2). Of the 57 investigations, four examined SRMH in a validation study, 37 used it as a major variable, and 16 included it as a minor variable. Seventeen studies used primary data collection, and the rest were secondary analyses of data from population health surveys. Most of the studies were cross-sectional, while six were prospective. The most commonly used surveys were the Canadian Community Health Survey (CCHS-13 studies), the National Latino and Asian American Study (NLAAS-9 studies) and the Medical Expenditure Panel Survey (MEPS-7 studies). Sixteen studies examined both SRMH and SRH.

Twenty-six studies were conducted in the United States, twenty in Canada, two in China, two in Singapore, and seven in other countries (Ukraine, Japan, England, Nigeria, Puerto Rico, Sri Lanka, and Turkey). Twenty-one studies did not report the ethnic composition of their sample, and the remaining looked at Asian Americans (Chinese, Filipino, Vietnamese, and 'other' Asian), Latino Americans (Mexican, Puerto-Rican, Cuban, and 'other' Latino), blacks, whites, 'non-whites', Turks, Sri Lankans and Nigerians. Twelve of these studies were specific to ethnic minorities, and nine made ethnic comparisons. Nine studies examined adults aged $60+$ and none examined children under the age of twelve. Nineteen studies were limited to individuals with psychological or psychiatric disorders, four examined individuals with other medical problems. 


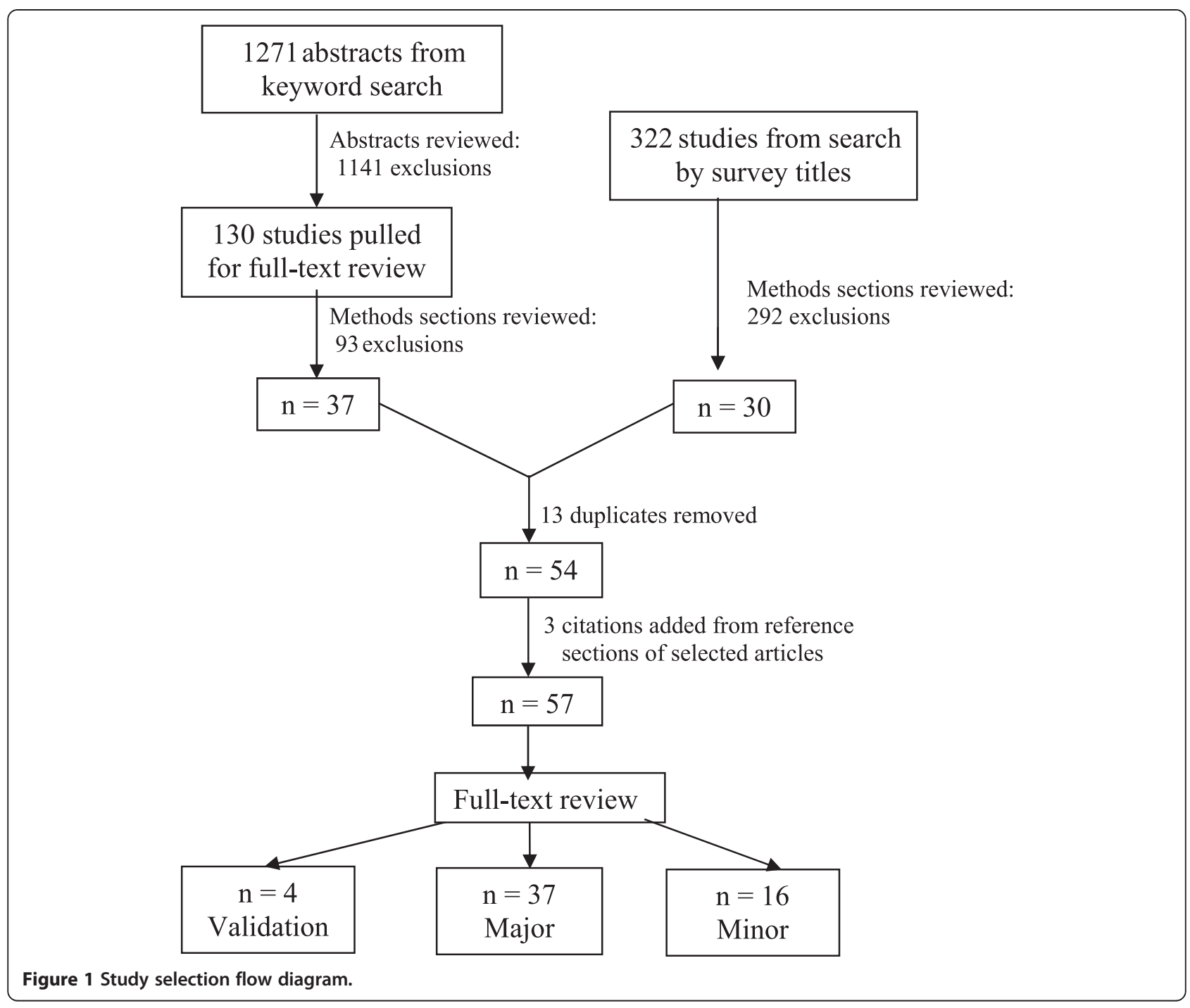

Sample sizes ranged from 121 to 120,559 . Seventeen studies had over 10, 000 participants. All investigations included men and women, except for one that looked only at men [37]. Thirteen studies did not report the proportion of males and females in their sample, thirteen conducted a gender-based analysis, and sixteen examined gender differences in SRMH.

\section{SRMH validation studies}

Four papers analyzed relationships between SRMH and clinical measures of mental health status. In 1997, Hoff et al. examined prospective data from the Epidemiologic Catchment Area study and found that high scores of SRMH were associated with decreased risk of major depression in the following year. Individuals with poor SRMH were 4.57 times more likely to have a major depressive episode than those with fair SRMH, and 9.97 times more likely than those with excellent SRMH. The effect held constant when controlling for age, gender, or a past history of depression. The authors concluded SRMH could be used to identify groups at higher risk for major depression, even in the absence of other risk factors [8]. Another study reported moderate correlation of SRMH with BASIS-24 [14]. In this study SRMH was a minor variable (see Additional file 1).

In 2007, Fleishman and Zuvekas analyzed MEPS data, a nationally representative US sample. They examined correlations between SRMH, SRH, physical and mental health subscales of the SF-12 health status survey, K6 scale of psychological distress, and Patient Health Questionnaire (PHQ-2) depression screener. The multi-item measures were more strongly correlated with each other $(r>.69)$ than with SRMH (correlations ranged between 0.33 and 0.49). SRH correlated more strongly with physical health subscales of the SF-12, and SRMH with mental health subscales of the SF-12, PHQ-2, and K6 [7]. Further, it is important to note that Fleishman and Zuvekas quantified the relationship between SRH and SRMH. They found 
Table 2 Self-rated mental health study characteristics

\begin{tabular}{|c|c|c|c|}
\hline Characteristic & \# of studies & Characteristic & \# of studies \\
\hline & $(\mathrm{N}=57)$ & & $(N=57)$ \\
\hline Country: & & Sample size: & \\
\hline United States & 26 & $<500$ & 8 \\
\hline Canada & 20 & $500-10,000$ & 32 \\
\hline Other ${ }^{*}$ & 11 & $>10,000$ & 17 \\
\hline Data type: & & *Study populations: & \\
\hline Primary data collection & 17 & Children $\leq 12$ yrs & 0 \\
\hline Secondary data analysis & 40 & Adults $60+$ & 9 \\
\hline Study design: & & Individuals with medical conditions ${ }^{\S}$ & 4 \\
\hline Cross sectional & 51 & Psychological or psychiatric disorders & 19 \\
\hline Prospective & 6 & Mental health service users & 5 \\
\hline \multirow[t]{3}{*}{ Case-control } & 1 & University students & 2 \\
\hline & & Caregivers for the elderly & 1 \\
\hline & & Veterans & 1 \\
\hline *Sex: & & General sample & 23 \\
\hline Mixed-sex sample & 56 & Objective for using SRMH: & \\
\hline Male-only sample & 1 & Validation study & 4 \\
\hline Female-only sample & 0 & Major variable in a research study & 37 \\
\hline Analysis of gender differences in SRMH & 16 & Minor variable in a research study & 16 \\
\hline Secondary data sources used in multiple studies: & & SRMH as major variable $\&$ review themes: & \\
\hline Canadian Community Health Survey & 13 & Validation/ Mental health condition & 9 \\
\hline Medical Expenditure Panel Survey & 7 & Physical health & 4 \\
\hline Mental Health Supplement to the Ontario Health Survey & 2 & Health service (utilization, satisfaction) & 13 \\
\hline National Latino Asian-American Study & 9 & Social determinants of health & 19 \\
\hline
\end{tabular}

*Categories under some headings are not mutually exclusive; column totals do not always add to 57.

${ }^{¥}$ Other: China 2, Singapore 2, England 1, Japan 1, Nigeria 1, Puerto Rico 1, Sri Lanka 1, Turkey 1, Ukraine 1.

${ }^{\S}$ Medical conditions: Asthma, medically unexplained physical symptoms, multiple sclerosis, restless leg.

Syndrome, hypertension.

both variables had a stronger association with each other $(r=.54)$ than with other physical and mental health scales. However, SRMH had unique associations with the mental health scales, even when adjusting for SRH. There may be some overlap between what these items are measuring, or a correlation exists between physical and mental health constructs [7].

In 2010, Mawani and Gilmour examined data collected by the CCHS 1.2, Mental Health and Well-Being. The results show consistent and strong association between poor/fair SRMH and several measures of mental morbidity which included WMH-CIDI, self-reported diagnosis of mental disorders, and level of stress. There was a gradient in mean scores of SRMH and prevalence of fair/poor mental health by recency of WMH-CIDI based mental disorders [38]. Jang et al. in 2012 examined a community sample of 420 Korean American older adults residing in the New York City. They found that SRMH was strongly associated with other mental health measures: CES-D $(r=.42)$, GDS-SF $(r=.42)$ and PHQ-9 $(\mathrm{r}=.50)$ while controlling for socio-demographics and physical health related variables. These three measures of depressive symptoms made significant contribution to SRMH. The variance explained by the short-form CES-D was $11 \%$, by the GDS-SF was $10 \%$, and by the PHQ-9 was 16\% [39].

\section{SRMH and mental health conditions}

Five studies examined SRMH as a major variable in relationship to mental health conditions. In 2006, Olfson conducted a prospective analysis of MEPS data and found that patients with fair or poor SRMH were almost two times more likely to be among the $26.7 \%$ of the sample who continued antidepressant therapy beyond 90 days. Results were adjusted for race, age, sex, and pre-treatment mental health status [11]. Study by Tiwari and Wang in 2006 found that Chinese participants were more likely than other Asians or whites to report fair or poor SRMH. However, Chinese and other Asians had lower prevalence rates of mental and substance use- 


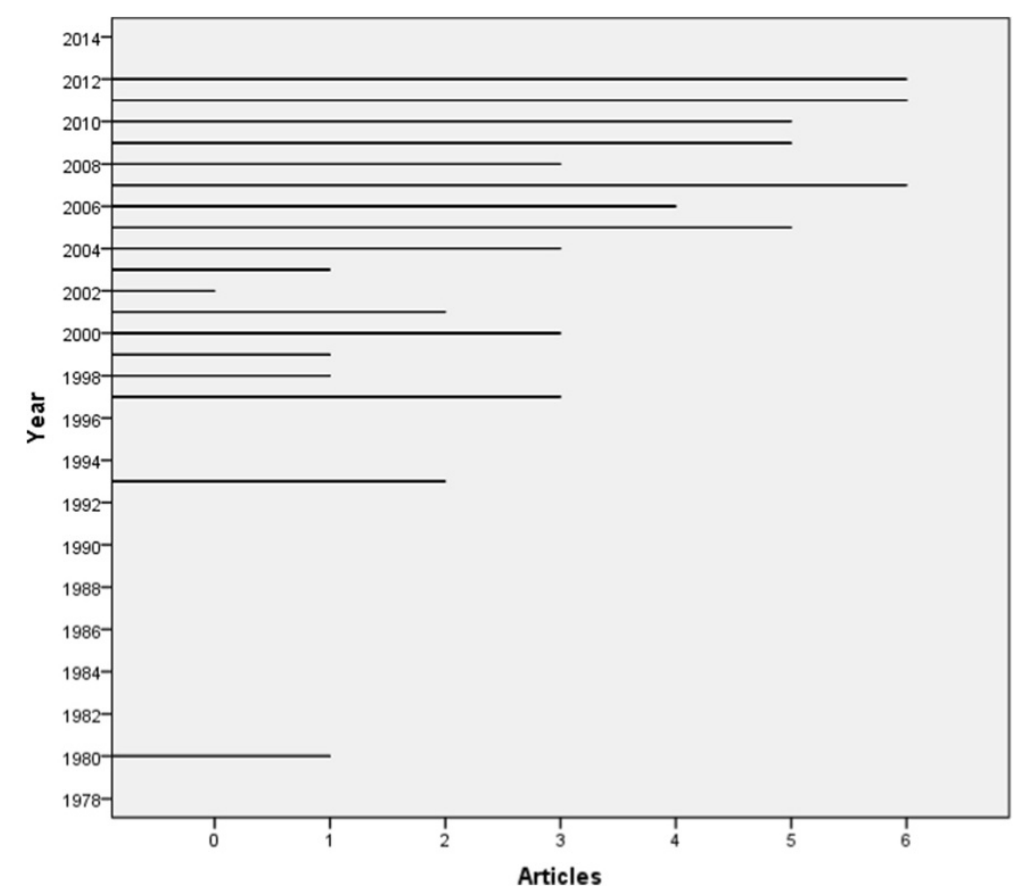

Figure 2 SRMH articles yearly since 1980.

related disorders [6]. In 2008, Zuvekas and Fleishman used MEPS data and compared Whites, Hispanics and Blacks. They found a weaker association between SRMH and emotional symptoms and SRMH and service use for Hispanics and Blacks compared to Whites. They suggested that ethnic differences exist in interpreting emotional symptoms and need for services [9]. In 2011, Kim et al. examined SRMH and psychiatric disorders among non-Hispanic Whites, Blacks, Hispanics, and Asians. They found that non-Hispanic Whites with poor SRMH were more likely to have mood and anxiety disorders [40]. Another study by Kim et al. in 2012 examined the associations between SRMH and diagnoses of psychiatric disorders among American subgroups of Chinese, Filipinos, and Vietnamese origin. The results show that, after controlling for covariates, SRMH was significantly associated with diagnoses for any 12-month DSM-IV psychiatric disorders only among Filipinos (AOR: 2.06; 95\% CI: $1.29-$ 3.32) [41]. Ethnicity seems to moderate the relationship between SRMH and mental health conditions.

\section{SRMH \& physical health}

Out of four studies using SRMH as a major variable, three examined relationships between poor physical health and fair or poor SRMH and one focused on the quality of life. In 2006, Dogra and Baker examined a group of asthmatics and found that physically active asthmatics had significantly greater SRMH and fewer chronic physical/mental chronic conditions [42]. In 2007, a descriptive study by Park and Knudson report that people with medically unexplained physical symptoms (MUPS) were more likely than those without MUPS to rate their mental health as fair or poor [43]. In 2011, El-Gabalawy examined data from the CCHS 1.2 and found that the comorbidity of anxiety with lung diseases resulted in poor SRMH after adjusting for confounding variables [44].

In 2010, Sawatzky et al. examined SRMH and self-rated physical health as key independent variables in predicting the quality of life (QOL) among Canadian adolescents. They included five life domains (satisfaction with family, friends, living environment, school and self) as mediating variables. The authors found that SRMH, and to a lesser degree self-reported physical health, was significantly associated with differences in satisfaction with five life domains and global QOL. The study also revealed that adolescents differentiated SRMH and self-rated physical health as distinct domains. SRMH was more strongly associated with depressive symptoms, measured by CES-D, than self-rated physical health. The latter was more strongly associated with physical activity than SRMH [12].

\section{SRMH and use of health services}

Thirteen studies examined SRMH as a major variable in relation to health services for its utilization, help-seeking and satisfaction. Of these thirteen, ten studies emphasized help seeking and service use, while three emphasized client satisfaction.

Studies on the utilization of health services examined the use of mental health services, complementary services 
and general practice in relation to SRMH. In 1997, Katz examined SRMH and the use of outpatient mental health care in Ontario and the US. The odds of receiving any medical or psychiatric or social services for persons with fair or poor SRMH were 2.7 in the US versus 5.0 in Ontario; the difference was eliminated on controlling the perceived need for care. Other control variables included sex, urban location, and age. SRMH was the second highest predictor of service use, preceded by presence of an affective disorder. Anxiety disorders, substance dependence, and comorbid mental health conditions were less predictive [45]. In 2001, Albizu-Garcia et al. examined mental care utilization prospectively among Puerto Ricans and found that poor SRMH and service need were stronger predictors of service use for men than for women [46]. Zuvekas and Flieshman (outlined previously), found fair or poor SRMH was predictive of ambulatory mental health visits and purchasing medications for mental health treatment. Analyses were controlled for sociodemographic factors, health insurance coverage, chronic physical health conditions, supply of psychiatrists with separate regressions including specific mental health scales [9]. The use of complementary services (e.g., chiropractic, acupuncture, massage) was examined by Druss and Rosenheck in 2000. They found the likelihood of using any complementary service did not change with poor or fair SRMH while controlling for age, sex, race, education, total medical/mental conditions, and region. Yet, presence of a mental condition was predictive of the use of complementary service in a similar multivariate model [36]. In 2007, Nabalamba and Miller reported that Canadians reporting fair or poor SRMH were more likely to visit a general practitioner or specialist. A significant association remained after adjusting for age, sex, ability to converse in English or French, household income, urban/rural residence, and having a family doctor [10]. In 2009, Vasiliadis examined CCHS 1.2 dataset for the determinants (grouped as need, enabling and predisposing factors) of visits to family physicians, psychiatrists, psychologists, psychotherapists, and other health professionals for mental health reasons. Among fifteen 'need based' significant predictors of service use, SRMH was fifth from the bottom with odds ratio of 1.37 [47]. In multivariable analyses of NLAAS data, Kim et al. in 2010 found that poor self-rated mental health was associated with significantly greater mental health service use among immigrants age 60 and older [48].

Similar findings have been reported by two studies conducted in Singapore. In multivariate analyses examining depressive and anxiety disorders, fair or poor SRMH and acknowledging having a mental illness were predictive of service use for mental and emotional health among adult Singaporeans; health beliefs and social support were not [49]. Another multivariate analysis of Singaporean elderly found fair or poor SRMH was an independent predictor of using mental health services [50].

The relationship between SRMH and help-seeking has been also reported. A descriptive analysis of mental health among Nigerian university students found SRMH was related with $\mathrm{SRH}$, neuroticism, and having problems to discuss with a doctor [2].

\section{SRMH and experiences with care}

Three studies examined SRMH and health service satisfaction. In 2000, Rohland et al. examined American Medicaid patients to determine the relationship between mental health service satisfaction, SRMH, and life satisfaction. The authors found correlations between all three variables for people with schizophrenia but not for those with affective, anxiety or adjustment disorders [51]. Follow-up study is needed to clarify the relationship between service satisfaction and SRMH amongst groups of differing diagnoses to identify whether other variables could be responsible for this effect. In 2007, Raleigh et al. in England found that people with fair or poor SRMH were less likely to be satisfied with mental health services. SRMH was the strongest predictor among all study variables (ethnicity, age, living alone, employment status, and hospital admissions) [52]. Eselius in 2008 found that evaluations of managed behavioural health plans varied by SRMH. Those with excellent or good SRMH gave higher ratings to the plan than those with fair or poor SRMH [53].

\section{SRMH and social determinants of health}

Nineteen studies examined SRMH as a major variable in relation to social determinants of health, such as socioeconomic status (e.g., education, income and type of employment), social environments (e.g., family support, community belonging, neighbourhood and nativity), age, gender or ethnicity/race.

Since 1990's studies have examined SRMH in relation to socioeconomic status along with age, gender and other demographics. In 1993, Yu and Wang examined social status and SRMH in a geriatric outpatient sample in China and found that people with very high and very low levels of education had lower SRMH than blue collar workers, civil servants, and white-collar workers. A potential explanation for low SRMH among highly educated individuals hypothesized to be the effect of the communist regime [54]. In 1997, Yu et al. analyzed this dataset to examine social factors in relation to $\mathrm{SRH}$ and SRMH. The predictors of low SRMH were old age, perceived lack of family respect, number of diseases, conflictual neighbourhood relations, percentage of income spent on rent, unmet preference to live with a son, and personal monthly income [55]. In 2000, Druss and 
Rosenheck found respondents with fair or poor SRMH were older, less likely to have a high school education, and had more self-reported mental or physical conditions [36]. In 2000, O'Donell examined SRMH among veterans and non-veterans and found lower SRMH for the latter but this group difference was eliminated on controlling for demographic, socioeconomic, and healthrelated factors [37]. In 2005, Cohen and Patten found a gender effect among Alberta medical residents; more males reported excellent SRMH than female residents. Overall $17 \%$ of residents reported fair or poor SRMH compared to $8 \%$ in national community health survey [56]. In 2006, Shields found that low satisfaction with job was related to fair or poor SRMH [57]. In 2008, Zuvekas and Flieshman found poorer SRMH among those who had a lower income, were less educated, female, or aged 41-60 [9]. In 2010, De Castro examined NLAAS data finding that employment frustration was associated with low SRMH even after controlling for gender, age, ethnicity, education, occupation, income, whether immigrated for employment, years in the United States, English proficiency, and a general measure for everyday discrimination [58]. In 2010, Maximova et al. examined resettlement experiences of Canadian refugees and found that having employment and access to settlement services were associated with improvements in SRMH while time spent in a refugee camp and having held a professional job in home country were associated with a decline in SRMH [59].

Some studies have examined the relationship between SRMH and the social environment. In 2005, Statistics Canada released a report on community belonging and self-perceived health using CCHS dataset. Stronger feelings of belonging were associated with substantially better SRMH and self-rated physical health [60]. In 2007, Mulvaney-Day et al. analyzed NLAAS dataset to examine the relationships between social support, social cohesion, and self-rated physical health and SRMH in a sample of Latinos. They found that family support was strongly associated with positive SRMH after controlling for language, education, income and other demographics [61]. In 2009, Zhang analyzed NLAAS data to examine the role of social connections (i.e. family cohesion, relative support, friend support, and neighborhood cohesion), socioeconomic status, and immigration-related factors on the self-rated physical and SRMH. The four types of social connections were all related to SRMH but family cohesion had independent and direct effects on SRMH over and above controls and mediators [62]. A multivariate analysis looking at neighbourhood environment and SRMH in Southern Sri Lanka found that environmental stressors (nuisance from neighbours or drug users, shortage of water, or having poor water/ sewage drainage system) were associated with fair or poor SRMH, but not with fair or poor $\mathrm{SRH}$ [63]. In 2012, three studies were published using
NLASS data, assessing the relationship between SRMH and nativity. Within a sample of first-generation Asian Americans, Lam et al. examined SRMH and the effects of age of immigration, age, and perceived difference on social status. There was no significant effect on SRMH due to age and age of immigration. However, when perceived difference in social status was considered, then age had a bearing on both physical and mental health [64]. Schachter et al. found that use of both English and ethnic language was associated with better self-rated physical health and SRMH; associations were partially mediated by socioeconomic status and family support [65]. In 2012, John et al. examined the associations of nativity and occupational class with SRMH while controlling for age and gender. Comparing U.S. born-Asians and immigrant Asians, John et al. found that immigrants were more likely to report fair or poor SRMH (adjusted OR 2.6) though less likely to report mental disorder and anxiety (adjusted odd ratio 0.6). No gradient was found between occupational class and SRMH within Asian immigrants, unlike the U.S. born Asians [66].

Several studies examined differences in SRMH associated with race and ethnicity. Using MEPS data, Zuvekas and Fleishman found Blacks and Hispanics were more likely than Whites to report excellent SRMH, and less likely to report poor SRMH (even when they had low scores on the mental component summary of the SF-12). In 2011, Veenstra examined data from Toronto and Vancouver. Associations were examined between SRMH, SRH and self-identified racial identities (i.e., Asian, Black, South Asian, and White). Respondents expressing Asian identity reported poorer SRMH and $\mathrm{SRH}$, which were not explained by their socio-economic status [67]. Additional studies examining ethnic and racial differences are described under theme of mental health conditions.

SRMH has also been examined in relationship to care giving or smoking. A prospective study of caregivers for the elderly found SRMH to have declined during the 2 -year study period. Decline in SRMH was predicted by poor baseline SRMH and decline in SRH [68]. A study of smoking among Nova Scotians used SRMH as a minor variable and found that more people with poor SRMH smoked compared to those with poor SRH [35].

\section{Discussion}

Our scoping review of the literature found 57 studies providing information on the performance of SRMH in diverse contexts. SRMH correlated moderately with mental health scales, but there are ethnic differences in responses to the item. Poor SRMH was associated with poor SRH, physical health problems, increased health service utilization, and a lower likelihood of being satisfied with mental health services. Some studies found age and gender disparities in SRMH but others did not. 
While few studies conducted formal validations of SRMH, its use in the literature gives us important information about its relationship with other variables.

This study is the first review of SRMH literature. Given the increasing use of this item in the recent years, it is important to understand the scientific contributions made by this item and the strengths and weaknesses in its use. We optimized the number of articles we found by using a broad, structured search strategy. However, a limitation of our research is that SRMH terminology is not yet standardized, and some studies may not have been captured by the selected keywords. We tried to minimize this limitation by conducting a second search of surveys known to include SRMH. A limitation of the SRMH research literature is that heterogeneity precludes meta-analyses. The review focused on single item SRMH, analogous to the single item SRH. Thus, studies that measure other aspects of mental health by using other single items were excluded.

The moderate correlations between SRMH and mental health scales indicate these measures are related but not interchangeable. SRMH may be measuring factors outside the scope of mental health scales, but based on current literature it is unclear what these other factors are. Given the similar wording and correlation between SRMH and SRH, we may be able to look toward SRH literature for hypotheses. When SRH was first studied, researchers found moderate correlations between SRH and current health status. When longitudinal studies were conducted, SRH was shown to be an equal or stronger predictor of mortality, morbidity, and utilization than many commonly used measures. Similarly, SRMH may be capturing developing mental health problems, in addition to existing disorders. Studies included in this scoping review have shown relationships between SRMH and health service utilization. Hoff et al. (1997) have demonstrated that SRMH can predict the risk of future depression. More investigation is needed to fully understand these relationships. While single items have the advantage of simplicity and ease of administration, they cannot capture complexities assessed using multi-item scales, and like other instruments may result in false positives and negatives. However, they can provide important information. The use of SRMH in epidemiologic and health surveys and the relationships identified in this review indicate that SRMH could become a robust population mental health measure. It has potential to provide estimates of the mental health of populations, be used to assess change over time in response to changes in policy and practice. SRMH may also hold value as a screener to identify individuals and populations at risk for future mental health problems, but research is limited thus far.

\section{Conclusions}

SRMH is seeing increased use in research and in population health surveys. This scoping review points to a number of relationships between SRMH and mental, physical, social, and utilization variables. SRMH may also be predictive of mental morbidity. However, more work needs to be done before these relationships can be firmly established. Future research should continue to further define the relationship between SRMH and measures of mental health or specific disorders. More longitudinal research is needed to determine whether SRMH is predictive of future mental health conditions. Studies should also look at how SRMH varies by sociodemographic characteristics (sex, ethnicity, age, socioeconomic status). In addition, more information is needed about how different population subgroups respond to this item, particularly if it is to be used to assess disparities. Finally, qualitative analysis could be useful in understanding individual response mechanisms behind this item.

\section{Additional file}

Additional file 1: Table 3. Studies Using Self-Rated Mental Health

(arranged thematically).

Competing interests

The authors declare that they have no competing interests.

\section{Authors' contributions}

All authors made significant contributions in the conception and design ( $A J_{t}$ $F A, A B \& D S)$; review of literature $(A J, F A \& M B)$; synthesis (AJ, FA, $A B \& M B)$; and interpretation (AJ, FA, $A B, D S \& M B$ ). All authors contributed in writing and/or critically reviewing the manuscript and approve of the submitted version.

\section{Acknowledgements}

This research was funded in part by the Project for an Ontario Women's Health Evidence-Based Report (POWER Study) which received funding from Echo: Improving Women's Health in Ontario, an agency of the Ontario Ministry of Health and Long-Term Care. The opinions, results and conclusions reported in this paper are those of the authors and are independent from the funding source. No endorsement by the Ontario Ministry of Health and Long-Term Care is intended or should be inferred.

\section{Author details}

${ }^{1}$ School of Health Policy and Management, Faculty of Health, York University, 4700 Keele Street, HNES 414, Ontario M3J 1P3, Canada. ${ }^{2}$ OPTIMUS | SBR, 30 Adelaide St. E, Suite 600, Toronto, ON M5C 3G8, Canada. ${ }^{3}$ Women's Health Program at University Health Network, University of Toronto, 200 Elizabeth St, Toronto M5G 2C4, Canada. ${ }^{4}$ School of Heath Policy and Management, Critical Disability Studies, Faculty of Health, York University, 4700 Keele Street, Ontario M3J 1P3, Canada. ${ }^{5}$ Lawrence S. Bloomberg Faculty of Nursing; Institute of Health Policy, Management and Evaluation; Dalla Lana School of Public Health and Department of Medicine, University of Toronto, Keenan Research Centre in the Li Ka Shing Knowledge Institute, St. Michael's Hospital, 30 Bond Street, Toronto, ON M5B 1 W8, Canada.

Received: 17 March 2014 Accepted: 9 September 2014 Published: 17 September 2014

\section{References}

1. Rohrer JE, Pierce JRJ, Blackburn C: Lifestyle and mental health. Prev Med 2005, 40:438-443.

2. Jegede RO: Personality and mental health characteristics of Nigerian university students. Acta Psychiatr Scand 1980, 61:67-80.

3. McMichael AJ, Hetzel BS: An epidemiological study of the mental health ofAustralian university students. Int J Epidemiol 1974, 3:125-134. 
4. Robins LN, Helzer JE, Croughan J, Ratcliff KS: National institute of mental health diagnostic interview schedule. Its history, characteristics, and validity. Arch Gen Psychiatry 1981, 38:381-389.

5. Wittchen H-U: Reliability and validity studies of the WHO-composite international diagnostic interview: a critical review. J Psychiatr Res 1994, 28:57-84

6. Tiwari SK, Wang J: The epidemiology of mental and substance use-related disorders among white, Chinese, and other Asian populations in Canada. Can J Psychiatry 2006, 51:904-912.

7. Fleishman JA, Zuvekas SH: Global self-rated mental health: associations with other mental health measures and with role functioning. Med Care 2007, 45:602-609.

8. Hoff RA, Bruce ML, Kasl SV, Jacobs SC: Subjective ratings of emotional health as a risk factor for major depression in a community sample. Br J Psychiatry 1997, 170:167-172.

9. Zuvekas SH, Fleishman JA: Self-rated mental health and racial/ethnic disparities in mental health service use. Med Care 2008, 46:915-923.

10. Nabalamba A, Millar WJ: Going to the doctor. Health Rep 2007, 18:23-35

11. Olfson M, Marcus SC, Tedeschi M, Wan GJ: Continuity of antidepressant treatment for adults with depression in the United States. Am J Psychiatry 2006, 163:101-108.

12. Sawatzky R, Pamela AR, Johnson JL, Kopec JA, Zumbo BD: Self-reported physical and mental health status and quality of life in adolescents: a latent variable mediation model. Health Qual Life Outcomes 2010, 8:e1-e11.

13. Eisen SV, Ranganathan G, Seal P, Spiro IIIA: Measuring clinically meaningful change following mental health treatment. J Behav Health Serv Res 2007, 34:272-289.

14. Eisen SV, Normand SL, Belanger AJ, Spiro A, Esch D: The revised behaviour and symptom identification scale (BASIS-R): reliability and validity. Med Care 2004, 42:1230-1241.

15. Streib GF, Schuman EA, Phillips BS: An analysis of the validity of health questionnaires. Soc Forces 1958, 36:223-232.

16. Idler EL, Kasl S: Health perceptions and survival: do global evaluations of health status really predict mortality? J Gerontol: Soc Sci 1991, 46:55-65.

17. Vasiliadis H-M, Lesage A, Adair C, Boyer R: Service use for mental health reasons: cross-provincial differences in rates, determinants, and equity of access. Can J Psychiatry 2005, 50:614-619.

18. Miilunpalo S, Vuori I, Oja P, Pasanen M, Urponen H: Self-rated health status as a health measure: the predictive value of self-reported health status on the use of physician services and on mortality in the working-age population. J Clin Epidemiol 1997, 50:517-528.

19. Manora O, Matthewsb S, Powerb C: Self-rated health and limiting longstanding illness: inter-relationships with morbidity in early adulthood. Int J Epidemiol 2001, 30:600-607.

20. Arksey H, O'Malley L: Scoping studies: towards a methodological framework. Int J Soc Res Methodol 2005, 8:19-25.

21. Rohrer JE, Arif A, Denison A, Young R, Adamson S: Overall self-rated health as an outcome indicator in primary care. J Eval Clin Pract 2007, 13:882-888.

22. Shields M: Social anxiety disorder: Beyond Shyness. Health Rep 2004, 15(Suppl):45-61.

23. Harman JS, Edlund MJ, Fortney JC, Kallas H: The influence of comorbid chronic conditions on the adequacy of depression care for older Americans. J Am Geriatr Soc 2005, 53:2178-2183.

24. Olfson M, Marcus SC: National patterns in antidepressant medication treatment. Arch Gen Psychiatry 2009, 66:848-856.

25. Morin CM, LeBlanc M, Belanger L, Ivers H, Merette C, Savard J: Prevalence of insomnia and its treatment in Canada. Can J Psychiatry 2011, 56:540-548

26. Tintle N, Bacon B, Kostyuchenko S, Gutkovich Z, Bromet EJ: Depression and its correlates in older adults in Ukraine. Int J Geriatr Psychiatr 2011, 26:1292-1299.

27. Sevim S, Dogu O, Camdeviren H, Bugdayci R, Sasmaz T, Kaleagasi H, Aral M, Helvaci I: Unexpectedly low prevalence and unusual characteristics of RLS in Mersin, Turkey. Neurology 2003, 61:1562-1569.

28. Peterson EW, Cho CC, Finlayson ML: Fear of falling and associated activity curtailment among middle aged and older adults with multiple sclerosis. Mult Scler 2007, 13:1168-1175.

29. Ohtsu T, Kaneita Y, Aritake S, Mishima K, Uchiyama M, Akashiba T, Uchimura N, Nakaji S, Munezawa T, Shimada N, Kokaze A, Ohida T: Preferable forms of relaxation for health promotion, and the association between recreational activities and self-perceived health. Acta Med Okayama 2012, $66: 41-51$.

30. Watkins AJ, Kligman EW: Attendance patterns of older adults in a health promotion program. Public Health Rep 1993, 108:86-90.

31. Lin $E$, Chan B, Goering P: Variations in mental health needs and fee-forservice reimbursement for physicians in Ontario. Psychiatr Serv 1998 49:1445-1451.

32. Vega WA, Kolody B, Guilar-Gaxiola S: Help seeking for mental health problems among Mexican Americans. J Immigr Health 2001, 3:133-140.

33. Bergeron $E$, Poirier $L$, Fournier $L$, Roberge $P$, Barrette $G$ : Determinants of service use among young Canadians with mental disorders. Can J Psychiatry 2005, 50:629-636.

34. Kim G, Aguado Loi CX, Chiriboga DA, Jang Y, Parmelee P, Allen RS: Limited english proficiency as a barrier to mental health service use: a study of Latino and Asian immigrants with psychiatric disorders. J Psychiatr Res 2011, 5:104-110

35. Nova Scotia Department of Health: Smoking in Nova Scotia. Canadian Community Health Survey Topics. 2004. Cycle 2.1, Report 1:1-6. Avialable online: http://novascotia.ca/dhw/publications/annual-statistical-reports/ cchs_smoking_2004.pdf.

36. Druss BG, Rosenheck RA: Use of practitioner-based complementary therapies by persons reporting mental conditions in the United States. Arch Gen Psychiatry 2000, 57(7):708-714

37. O'Donnell JC: Military service and mental health in later life. Mil Med 2000, 165:219-223.

38. Mawani F, Gilmour $\mathrm{H}$ : Validation of self-rated mental health. Health Rep 2010, 21:e1-e15.

39. Jang Y, Park NS, Kim G, Kwag KH, Roh S, Chiriboga DA: The association between self-rated mental health and symptoms of depression in Korean American older adults. Aging Ment Health 2012, 16:481.

40. Kim G, DeCastro J, Chiriboga DA, Jang Y, Allen RS, Parmelee P: Associations between self-rated mental health and psychiatric disorders among older adults: do racial/ethnic differences exist? Am J Geriatr Psychiatry 2011, 19:416-422.

41. Kim G, Chiriboga DA, Bryant A, Huang C-H, Crowther M, Ma GX: Self-rated mental health among Asian American adults: association with psychiatric disorders. Asian Am J Psychol 2012, 3:44-52.

42. Dogra S, Baker J: Physical activity and health in Canadian asthmatics. J Asthma 2006, 43:795-799.

43. Park J, Knudson S: Medically unexplained physical symptoms. Health Rep 2007, 18:43-47.

44. El-Gabalawy R, Mackenzie CS, Shooshtari S, Sareen J: Comorbid physical health conditions and anxiety disorders:a population-based exploration of prevalence and health outcomes among older adults. Gen Hosp Psychiatry 2011, 33:556-564.

45. Katz SJ, Kessler RC, Frank RG, Leaf P, Lin E, Edlund M: The use of outpatient mental health services in the United States and Ontario: the impact of mental morbidity and perceived need for care. Am J Public Health 1997, 87:1136-1143.

46. Albizu-Garcia CE, Alegria M, Freeman D, Vera M: Gender and health services use for a mental health problem. Soc Sci Med 2001, 53:865-878

47. Vasiliadis H-M, Tempier R, Lesage A, Kates N: General practice and mental health care: determinants of outpatient service use. Can J Psychiatry 2009, 54:468-476

48. Kim G, Jang Y, Chiriboga DA, Ma GX, Schonfeld L: Factors associated with mental health service use in Latino and Asian immigrant elders. Aging Ment Health 2010, 14:535-542.

49. Ng TP, Jin AZ, Ho R, Chua HC, Fones CSL, Lim L: Health beliefs and help seeking behaviour for depressive and anxiety disorders among urban Singaporean adults. Psychiatr Serv 2008, 59:105-108

50. Nyunt MSZ, Chiam PC, Kua EH, Ng TP: Determinants of mental health service use in the national mental health survey of the elderly in Singapore. Clin Pract Epidemiol Ment Health 2009, 5:e1-e9.

51. Rohland BM, Langbehn DR, Rohrer JE: Relationship between service effectiveness and satisfaction among persons receiving Medicaid mental health services. Psychiatr Serv 2000, 51:248-250.

52. Raleigh VS, Irons R, Hawe E, Scobie S, Cook A, Reeves R, Petruckevitch A, Harrison J: Ethnic variations in the experiences of mental health service users in England. Results of a national patient survey programme. Br J Psychiatry 2007, 191:304-312. 
53. Eselius LL, Cleary PD, Zaslavsky AM, Huskamp HA, Busch SH: Case-mix adjustment of consumer reports about managed behavioural health care and health plans. Health Serv Res 2008, 43:2014-2032.

54. Yu LC, Wang M: Social status, physical, mental health, well-being and self-evaluation of elderly in China. J Cross-Cult Gerontol 1993, 8:147-159.

55. Yu LC, Zhang AY, Draper P, Kassab C, Miles T: Cultural correlates of self perceived health status among Chinese elderly. J Cross-Cult Gerontol 1997, 12:73-89.

56. Cohen JS, Patten S: Well-being in residency training: a survey examining resident physician satisfaction both within and outside of residency training and mental health in Alberta. BMC Med Educ 2005, 5:1-11.

57. Shields M: Unhappy on the job. Health Rep 2006, 17:32-37.

58. de Castro AB, Rue T, Takeuchi DT: Associations of employment frustration with self-rated physical and mental health among Asian American immigrants in the U.S. labor force. Public Health Nurs 2010, 27:492-503.

59. Maximova K, Krahn H: Health status of refugees settled in Alberta: changes since arrival. Can J Public Health 2010, 101:322-326.

60. Statistics Canada. Community belonging and self-perceived health: Early CCHS findings (January to: Your Community, Your Health: Findings from the Canadian Community Health Survey 2005. Health Stat Div: Minsit Ind Catalogue 2005, 82-621:1-24.

61. Mulvaney-Day NE, Alegria M, Sribney W: Social cohesion, social support, and health among Latinos in the United States. Soc Sci Med 2007, 64:477-495.

62. Zhang W, Ta VM: Social connections, immigration-related factors, and self-rated physical and mental health among Asian Americans. Soc Sci Med 2009, 68:2104-2112.

63. Perera B, Ostbye T, Jayawardana C: Neighbourhood environment and self-rated health among adults in southern Sri Lanka. Int J Environ Res Publ Health 2009, 6:2102-2112.

64. Lam J, Yip T, Gee G: The physical and mental health effects of age of immigration, age, and perceived difference in social status among first generation Asian Americans. Asian Am J Psychol 2012, 3:29-43.

65. Schachter A, Kimbro RT, Gorman BK: Language proficiency and health status: are bilingual immigrants healthier? J Health Soc Behav 2012, 53:124-145

66. John DA, de Castro AB, Martin DP, Duran B, Takeuchi DT: Does an immigrant health paradox exist among Asian Americans? Associations of nativity and occupational class with self-rated health and mental disorders. Soc Sci Med 2012, 75:2085-2098.

67. Veenstra G: Mismatched racial identities, colourism, and health in Toronto and Vancouver. Soc Sci Med 2011, 73:1152-1162.

68. Haug MR, Ford AB, Stange KC, Noelker LS, Gaines AD: Effect of giving care on caregivers' health. Res Aging 1999, 21:515-538.

\section{doi:10.1186/1472-6963-14-398}

Cite this article as: Ahmad et al: Single item measures of self-rated mental health: a scoping review. BMC Health Services Research 2014 14:398.

\section{Submit your next manuscript to BioMed Central and take full advantage of:}

- Convenient online submission

- Thorough peer review

- No space constraints or color figure charges

- Immediate publication on acceptance

- Inclusion in PubMed, CAS, Scopus and Google Scholar

- Research which is freely available for redistribution 